DOI: $10.17516 / 1997-1397-2021-14-4-404-413$

УДК 532.5.013.3

\title{
Solution of a Two-Layer Flow Problem with Inhomogeneous Evaporation at the Thermocapillary Interface
}

\author{
Victoria B. Bekezhanova* \\ Institute of Computational Modelling SB RAS \\ Krasnoyarsk, Russian Federation \\ Olga N. Goncharova ${ }^{\dagger}$ \\ Altai State University \\ Barnaul, Russian Federation \\ Ilya A. Shefer $\ddagger$ \\ Siberian Federal University \\ Krasnoyarsk, Russian Federation
}

Received 27.01.2021, received in revised form 17.03.2021, accepted 05.04.2021

\begin{abstract}
The Ostroumov-Birikh type exact solution of thermodiffusion convection equations is constructed in the frame of mathematical model considering evaporation through the liquid-gas interface and the influence of direct and inverse thermodiffusion effects. It is interpreted as a solution describing steady flow of evaporating liquid driven by co-current gas-vapor flux on a working section of a plane horizontal channel. Functional form of required functions is presented. An algorithm for finding all the constants and parameters contained in the solution is outlined, and their explicit expressions are written. The solution is derived for the case of vapor absorption on the upper wall of the channel which is set with the help of the first kind boundary condition for the function of vapor concentration. Applicability field of the solution is briefly discussed.
\end{abstract}

Keywords: mathematical model, boundary value problem, exact solution, evaporative convection

Citation: V.B. Bekezhanova, O.N. Goncharova, I.A. Shefer, Solution of a Two-Layer Flow Problem with Inhomogeneous Evaporation at the Thermocapillary Interface, J. Sib. Fed. Univ. Math. Phys., 2021, 14(4), 404-413. DOI: 10.17516/1997-1397-2021-14-4-404-413.

\section{Introduction}

The widespread use of two-phase systems in different technologies motivates the intensive development of the experimental and theoretical methods for studying the features of convective flows accompanied by evaporation in the frame of various approaches [1]. Examples of such technologies are the fluidic cooling, thermal coating or drying processes etc. Full-scale experimental elaboration and testing of the real technological systems can be very expensive and sometimes impossible (for example, if it is expected that these systems will be used in microgravity). Thus, preliminary theoretical investigation based on the mathematical modeling is the necessary requirement and an indispensable part when solving the optimization problems of fluid technologies and in the search for innovative technical solutions.

\footnotetext{
*vbek@icm.krasn.ru https://orcid.org/0000-0003-2068-6364

${ }^{\dagger}$ gon@math.asu.ru https://orcid.org/0000-0002-9876-4177

${ }^{\ddagger}$ ishefer@sfu-kras.ru https://orcid.org/0000-0003-0923-9352

(c) Siberian Federal University. All rights reserved
} 
Currently, mathematical models built on the basis of the Navier-Stokes and heat transfer equations or their approximations are the most widely used ones for theoretical investigations of the processes in the two-phase systems. These equations are the results of symmetry of the space-time, i. e. the fulfilment of the fundamental conservation laws was implied in deriving the equations. This fact enables to obtain the significant results in the study the problems of the fluid flows with heat and mass transfer at the thermocapillary interfaces. We focus on the search and investigation of an exact solution of the governing system of differential equations, since the solution inherit basic properties of symmetry of the space-time and of a fluid moving in the space, thereby ensuring feasibility of physical processes described by this solution.

Among possible solutions of the evaporative convection problems are especially highlighted the Ostroumov-Birikh type solutions [1]. They take into account the presence of temperature gradient which can appear both due to evaporation and applied outside or interfacial thermal load. The applicability of such a class of solutions for describing the two-layer flows with diffusive type evaporation at the interface in a horizontal channel is confirmed by a good qualitative agreement between the experimental data and theoretical results [2]. The specific feature of these solutions is that they allow one to test various types of boundary conditions for the vapor concentration and temperature functions, to correctly take into account the influence of the external controlling actions (thermal, mechanical, fluid flow rate etc.) as well as the gravity and thermodiffusion effects [3,4].

For the first time, the problem of unidirectional two-layer flows induced by the gravity and Marangoni forces was considered in [5]. The first results of the study the flows with evaporation in a bilayer system based on an analogue of the Ostroumov-Birikh solution were presented in [6]. $2 \mathrm{D}$ and $3 \mathrm{D}$ generalizations of the solution obtained in the framework of the evaporative convection problem in the liquid - gas system with the sharp interface admitting the phase transition were constructed in $[7,8]$. The uniform character of evaporation was considered in all the listed works.

In the present paper, an exact solution of the convection equations to describe joint flow of the evaporating liquid and gas-vapor mixture in a horizontal minichannel under conditions of the given gas flow rate and full vapor absorption on the upper channel boundary is constructed. The aim of this work is to take into account an inhomogeneous with respect to the longitudinal coordinate character of evaporation at the interface.

\section{Problem statement and form of exact solution}

The stationary flow of two viscous incompressible media (of the liquid and gas-vapor mixture) filling the plane channel and having the common thermocapillary interface $\Gamma$ is considered (Fig. 1). In the Cartesian coordinate system $(x, y)$ the gravity acceleration vector $\mathbf{g}$ has the coordinates $\mathbf{g}=(0,-g)$. The upper and lower boundaries of the channel $y=h$ and $y=-l$ are the rigid walls. The interface remains to be flat, it is given by the equation $y=0$.

The Oberbeck-Boussinesq approximation of the Navier-Stokes equations is used to describe the flow in each phase. In two-dimensional case the constitutive equations have the following form:

$$
\begin{gathered}
u \frac{\partial u}{\partial x}+v \frac{\partial u}{\partial y}=-\frac{1}{\rho} \frac{\partial p}{\partial x}+\nu\left(\frac{\partial^{2} u}{\partial x^{2}}+\frac{\partial^{2} u}{\partial y^{2}}\right) \\
u \frac{\partial v}{\partial x}+v \frac{\partial v}{\partial y}=-\frac{1}{\rho} \frac{\partial p}{\partial y}+\nu\left(\frac{\partial^{2} v}{\partial x^{2}}+\frac{\partial^{2} v}{\partial y^{2}}\right)+g(\beta T+\gamma C) \\
-405-
\end{gathered}
$$




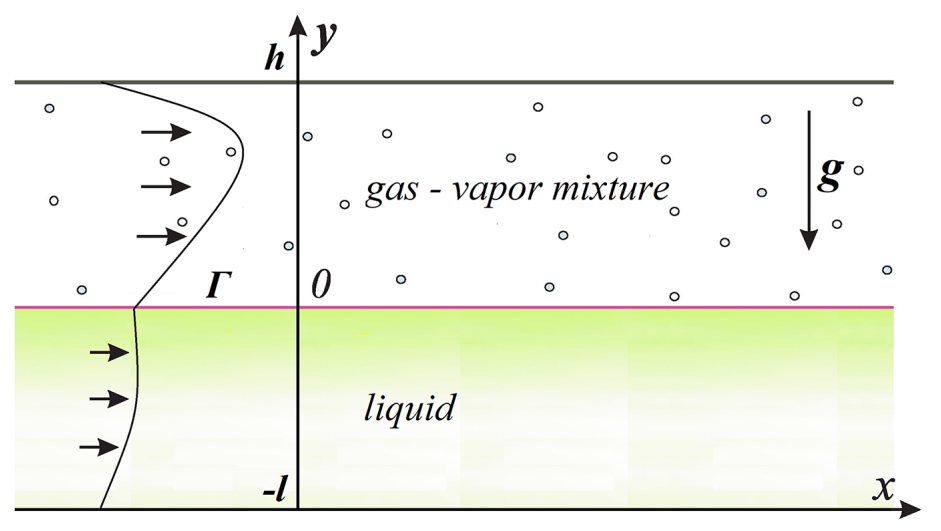

Fig. 1. Flow scheme.

$$
\begin{gathered}
\frac{\partial u}{\partial x}+\frac{\partial v}{\partial y}=0 \\
u \frac{\partial T}{\partial x}+v \frac{\partial T}{\partial y}=\chi\left(\frac{\partial^{2} T}{\partial x^{2}}+\frac{\partial^{2} T}{\partial y^{2}}+\delta\left(\frac{\partial^{2} C}{\partial x^{2}}+\frac{\partial^{2} C}{\partial y^{2}}\right)\right)
\end{gathered}
$$

The vapor transfer in the gas phase is governed by the convective diffusion equation, which is the result of the Fick's law [9]:

$$
u \frac{\partial C}{\partial x}+v \frac{\partial C}{\partial y}=D\left(\frac{\partial^{2} C}{\partial x^{2}}+\frac{\partial^{2} C}{\partial y^{2}}+\alpha\left(\frac{\partial^{2} T}{\partial x^{2}}+\frac{\partial^{2} T}{\partial y^{2}}\right)\right)
$$

The terms $\gamma C$ in (2.2) and $\delta \Delta C$ in (2.4) are taken into account by modeling of flows in the gas-vapor layer. In equations (2.1)-(2.5) the following notations are used: $u, v$ are the longitudinal and transversal components of the velocity vector, $p$ is the deviation of pressure from the hydrostatic one, $T$ is the temperature, $C$ is the vapor concentration in background gas, $\rho$ is the density of the liquid and gas (some reference value of the density), $\nu$ is the kinematic viscosity coefficient, $\chi$ is the heat diffusivity coefficient, $D$ is the coefficient of vapor diffusion in the gas, $\beta$ is the coefficient of thermal expansion, $\gamma$ is the concentration coefficient of density, the parameters $\delta$ and $\alpha$ characterize the Dufour and Soret effects (the effects of diffusive thermal conductivity and thermodiffusion, correspondingly) [10].

Let the exact solution of the governing equations (2.1)-(2.5) be of a special type, when only the longitudinal velocity component is not equal to zero and depends on the transverse coordinate; functions of temperature and vapor concentration have the linear components with respect to the longitudinal coordinate:

$$
\begin{gathered}
u_{i}=u_{i}(y), \quad v_{i}=0, \quad T_{i}=T_{i}(x, y)=\left(a_{1}^{i}+a_{2}^{i} y\right) x+\vartheta_{i}(y) \\
C=C(x, y)=\left(b_{1}+b_{2} y\right) x+\phi(y), \quad p_{i}=p_{i}(x, y)
\end{gathered}
$$

Index $i$ denotes characteristics of corresponding fluid: $i=1$ relates to the liquid in the lower layer, $i=2$ regards to the gas-vapor mixture filling the upper layer. Parameters $a_{2}^{i}, b_{j}(i=1,2$; $j=1,2$ ) are the constants, their values will be determined with the help of boundary conditions. Furthermore, the boundary conditions will dictate certain linking relations for the parameters. 


\section{Boundary conditions}

The boundary conditions will be written subject to the form of exact solution (2.6) of equations (2.1)-(2.5). The no-slip conditions are fulfilled on the fixed impermeable channel walls

$$
u_{1}(-l)=0, \quad u_{2}(h)=0,
$$

and the linear temperature distribution is prescribed on these walls

$$
T_{1}(x,-l)=A_{1} x+\vartheta^{-}, \quad T_{2}(x, h)=A_{2} x+\vartheta^{+} .
$$

The condition for vapor concentration on the upper wall is determined by the property of this wall to instantaneously completely absorb the vapor:

$$
C(x, h)=0 .
$$

In some real physical cases the vapor absorbtion property is confirmed by a possibility of a freezing out of the vapor. The applicability of boundary condition of such a type in this problem in frame of 3D statement is discussed in [11].

On the thermocapillary interface $\Gamma$ given by the equation $y=0$ the kinematic and dynamic conditions as well as the condition of heat balance should be set [12]. The kinematic condition is fulfilled automatically in view of the form of the velocity vector components (see (2.6)). Projection of the dynamic condition on the unit tangential vector to the interface is written as follows:

$$
\rho_{1} \nu_{1} u_{1 y}=\rho_{2} \nu_{2} u_{2 y}-\left.\sigma_{T} T_{x}\right|_{y=0},
$$

where $\sigma_{T}>0$ is the temperature coefficient of the surface tension $\sigma$ which linearly depends on the temperature, $\sigma=\sigma_{0}-\sigma_{T}\left(T-T_{0}\right), \sigma_{0}>0$ is the characteristic value of the surface tension at a relative temperature $T_{0}$. Projection of the dynamic condition on the unit normal vector to the interface leads to the equality

$$
p_{1}=p_{2} .
$$

We demand the fulfilment of continuity conditions for the tangential component of velocity vector and temperature at the interface:

$$
u_{1}=u_{2}, \quad T_{1}=T_{2} .
$$

The continuity of normal component of the velocity vector ensues from the kinematic condition.

The heat transfer condition and the mass balance equation are stated as follows:

$$
\begin{gathered}
\kappa_{1} T_{1 y}-\kappa_{2} T_{2 y}-\left.\delta \kappa_{2} C_{y}\right|_{y=0}=-\lambda M, \\
M=-D \rho_{2}\left(C_{y}+\left.\alpha T_{2 y}\right|_{y=0}\right) .
\end{gathered}
$$

The relations include the effects of the thermodiffusion and duffusive thermal conductivity characterized by coefficients $\alpha$ and $\delta ; \lambda$ is the latent heat of evaporation. In the present paper, the exact solution is constructed under assumption, that the evaporation mass flow rate of the liquid at the interface linearly depends on the longitudinal coordinate:

$$
M=M(x)=M_{0}+M_{x} x .
$$

The presupposition implies that nonuniform (inhomogeneous) character of phase transition is examined. The evaporation mass flow rate $M$ is one of the important characteristics of the 
evaporative convection. The positive values of $M$ correspond to evaporation of the liquid into the gas flow; the negative values regards to the vapor condensation.

The saturated vapor concentration is defined with the help of the relation being a sequence of the Clapeyron-Clausius and Mendeleev-Clapeyron equations $[6,7,13]$ :

$$
\left.C\right|_{y=0}=C_{*}\left[1+\varepsilon\left(\left.T_{2}\right|_{y=0}-T_{0}\right)\right]
$$

In this equation $\varepsilon=\lambda \mu_{0} /\left(R T_{0}^{2}\right), \mu_{0}$ is the molar mass of the evaporating liquid, $R$ is the universal gas constant, $C_{*}$ is the saturated vapor concentration at $T_{2}=T_{0}$. Equation (3.10) is valid under assumption of smallness of the dimensionless parameter $\varepsilon T_{*}\left(T_{*}\right.$ is a characteristic temperature drop), that is provided by moderate values of temperature and temperature drops.

To close the problem statement the condition of a given gas flow rate is assumed to be satisfied:

$$
Q=\int_{0}^{h} \rho_{2} u_{2}(y) d y
$$

Used form of the boundary conditions allows one to correctly describe the phase transition of diffusive type. Thus, boundary-value problem (2.1)-(2.5), (3.1)-(3.11) presents the mathematical model to simulate convection in multiphase system under conditions of weak evaporation.

\section{The class of the exact solutions}

The fulfilment of condition of temperature continuity (3.6) at the interface dictates the following equality: $a_{1}^{i}=A(i=1,2)$. The value determines the longitudinal temperature gradient presetting the intensity thermal effects on the interface, and as consequence, the intensity of evaporation and surface tension-driven convection.

Deriving the solution of equations (2.1)-(2.5) in the form (2.6) results in the explicit expressions for the required functions which define basic characteristics of the bilayer system (velocity $u_{i}$, pressure $p_{i}$, temperature $T_{i}$ in $i$-th phase and vapor concentration $C$ in gas layer):

$$
\begin{gathered}
u_{i}(y)=c_{3}^{i}+c_{2}^{i} y+c_{1}^{i} \frac{y^{2}}{2}+L_{3}^{i} \frac{y^{3}}{6}+L_{5}^{i} \frac{y^{4}}{24}, \\
p_{i}(x, y)=\left(d_{1}^{i}+d_{2}^{i} y+d_{3}^{i} \frac{y^{2}}{2}\right) x+c_{8}^{i}+K_{1}^{i} y+K_{2}^{i} \frac{y^{2}}{2}+K_{3}^{i} \frac{y^{3}}{3}+K_{4}^{i} \frac{y^{4}}{4}+ \\
+K_{5}^{i} \frac{y^{5}}{5}+K_{6}^{i} \frac{y^{6}}{6}+K_{7}^{i} \frac{y^{7}}{7}+K_{8}^{i} \frac{y^{8}}{8}, \\
T_{i}(x, y)=\left(A+a_{2}^{i} y\right) x+c_{5}^{i}+c_{4}^{i} y+N_{2}^{i} \frac{y^{2}}{2}+N_{3}^{i} \frac{y^{3}}{6}+N_{4}^{i} \frac{y^{4}}{24}+N_{5}^{i} \frac{y^{5}}{120}+N_{6}^{i} \frac{y^{6}}{720}+N_{7}^{i} \frac{y^{7}}{1008} \\
C(x, y)=\left(b_{1}+b_{2} y\right) x+c_{7}+c_{6} y+S_{2} \frac{y^{2}}{2}+S_{3} \frac{y^{3}}{6}+S_{4} \frac{y^{4}}{24}+S_{5} \frac{y^{5}}{120}+S_{6} \frac{y^{6}}{720}+S_{7} \frac{y^{7}}{1008} .
\end{gathered}
$$

Coefficients $L_{4}^{i}, L_{3}^{i}, S_{j}, K_{m}^{i}(i=1,2 ; j=2, \ldots, 7 ; m=1, \ldots, 8)$ are expressed by physical parameters of the problem $g, \beta_{i}, \nu_{i}, \chi_{i}, \rho_{i}, D, \gamma$, coefficients defining the longitudinal temperature and vapor concentration gradients $A, a_{2}^{i}, b_{i}(i=1,2)$, and by integration constants $c_{j}^{i}(i=1,2$; $j=1, \ldots, 5 ; 8), c_{6}, c_{7}$. Exact representations of the listed coefficients are given in Appendix. 


\section{The common scheme for finding the governing parameters and integration constants}

Implementation of boundary conditions (3.1)-(3.11) will lead to a system of equations for calculation of the integration constants $c_{j}^{i}(i=1,2 ; j=1, \ldots, 5 ; 8), c_{6}, c_{7}$. Determining these constants, the velocity and temperature profiles, the pressure distributions for both fluids and the vapor concentration in the gas are calculated with the help of formulas (3.12).

Below, the algorithm for finding all the unknown parameters and constants is outlined. Let the gas flow rate (3.11) and certain values of the longitudinal temperature gradients $A, A_{1}$ (see expression for the temperature functions in (3.12) and boundary conditions (3.2) be given.

(i) In the consequence of the heat transfer and mass balance conditions (3.7), (3.8) at the interface a relationship relating the longitudinal temperature gradients $A, A_{1}$ and $A_{2}$ is derived. It should be noted that both boundary gradients $A_{1}$ and $A_{2}$ can be given, then the corresponding relation to calculate the interfacial gradient $A$ is obtained.

(ii) Parameters $b_{1}, b_{2}, M_{x}$ characterizing the flow regime (2.6), (3.9) with nonuniform evaporation are determined with the help of $A, A_{2}$ on the basis of (3.3), (3.8), (3.10).

(iii) Solving the system of the linear algebraic equations being a consequence of the no-slip conditions (3.1), dynamic conditions (3.4), (3.5), condition of velocity continuity (3.6) and equality (3.11) defining the gas flow rate, the values of the unknowns $\left\{c_{1}^{i}, c_{2}^{i}, c_{3}^{i}\right\}(i=1,2)$ are calculated.

(iv) Conditions determining the thermal and vapor concentration boundary regimes (3.2), (3.3), conditions at interface setting the temperature continuity (3.6) and saturated vapor concentration (3.10), and heat balance equation (3.7) lead to the system of the linear algebraic equations for calculation $\left\{c_{4}^{2}, c_{5}^{2}, c_{6}\right\}$ and $\left\{c_{4}^{1}, c_{5}^{1}, c_{7}\right\}$.

(v) The value of $M_{0}$ will be computed with the help of obtained values $c_{4}^{2}$ and $c_{6}$.

Following this algorithm, all the required functions of the form (2.6) and the mass evaporation rate at the interface $M=M(x)$ in the form (3.9) are determined.

\section{Concluding remarks with regard to conditions of applicability of the solution}

To use the obtained solution for describing convection with evaporation in real physical systems, it should define conditions ensuring the correct application of the approach based on the utilization of the exact solutions of the fluid mechanics equations in the Oberbeck-Boussinesq approximation.

First of all, it must be remembered that the principal limitation for the use of the OberbeckBoussinesq approximation is to consider the heat and mass transfer processes occurring under moderate temperature drops. The equations of thermal-concentration convection written in form (2.1)-(2.4) present the "diffusive" laws of the transfer of mass, momentum and energy which adequately govern these processes near the thermodynamical equilibrium state. The moderate temperature drops, in turn, result in small variations of concentration. The latter ensures the correct using of the Fick's law written in form of convection-diffusion equation (2.5) and interface 
boundary condition (3.10). These requirements concerning quantitative changes in temperature and concentration in the system allow one to consider the processes of phase transition as the diffusive ones, and consequently, to believe that we deal with "weak" evaporation. It means that the phase transitions induced by critical thermal loads as, for example, while boiling are not considered.

The second point is to regard the flows with small velocities. It allows one to suppose that the gas in two-phase systems under study is an incompressible medium. It is worth to noting that for the mini- and microscale fluidic systems this assumption is quite justified [14]. Simultaneously, the requirement concerning the scale of the system is the condition when the Ostroumov-Birikh type solution gives plausible description of all the basic characteristics for a two-phase system with evaporation through the sharp interface [6].

Finally, taking into account the character of dependence of the temperature and vapor concentration functions on the longitudinal coordinate $x$ specified in (2.6), one can conclude that these functions will grow with growth of $x$. Then, according to the given physical interpretation of solution (2.6), it will give appropriate (physically feasible) results if the convective regimes are considered in the domain of finite size. One should control the values of $C$ function; they cannot be more than 1 , since we treat this function as mass fraction of the evaporating component in the background gas. If its values becomes more than 1 , it will immediately mean, that the solution gives "purely mathematical solution" of the boundary-value problem under consideration.

\section{Appendix. Formulas for calculating the coefficients in expressions (3.12)}

Coefficients $L_{4}^{i}, L_{3}^{i}$ :

$$
L_{4}^{1}=\frac{g \beta_{1} a_{2}^{1}}{\nu_{1}}, \quad L_{3}^{1}=\frac{g \beta_{1} A}{\nu_{1}}, \quad L_{4}^{2}=\frac{g}{\nu_{2}}\left(\beta_{2} a_{2}^{2}+\gamma b_{2}\right), \quad L_{3}^{2}=\frac{g}{\nu_{2}}\left(\beta_{2} A+\gamma b_{1}\right) .
$$

Coefficients $N_{7}^{i}, N_{6}^{i}, N_{5}^{i}, N_{4}^{i}, N_{3}^{i}, N_{2}^{i}$ :

$$
\begin{gathered}
N_{7}^{1}=\frac{g \beta_{1}\left(a_{2}^{1}\right)^{2}}{\nu_{1} \chi_{1}}, \quad N_{6}^{1}=5 \frac{g \beta_{1} A a_{2}^{1}}{\nu_{1} \chi_{1}}, \quad N_{5}^{1}=\frac{1}{\chi_{1}}\left(\frac{g \beta_{1}(A)^{2}}{\nu_{1}}+3 a_{2}^{1} c_{1}^{1}\right), \\
N_{4}^{1}=\frac{1}{\chi_{1}}\left(A c_{1}^{1}+2 a_{2}^{1} c_{2}^{1}\right), \quad N_{3}^{1}=\frac{1}{\chi_{1}}\left(A c_{2}^{1}+a_{2}^{1} c_{3}^{1}\right), \quad N_{2}^{1}=\frac{A}{\chi_{1}} c_{3}^{1}, \quad N_{7}^{2}=B_{2} \frac{g}{\nu_{2}}\left(\beta_{2} a_{2}^{2}+\gamma b_{2}\right), \\
N_{6}^{2}=\frac{g}{\nu_{2}}\left[B_{1}\left(\beta_{2} a_{2}^{2}+\gamma b_{2}\right)+4 B_{2}\left(\beta_{2} A+\gamma b_{1}\right)\right], \quad N_{5}^{2}=B_{1} \frac{g}{\nu_{2}}\left(\beta_{2} A+\gamma b_{1}\right)+3 B_{2} c_{2}^{1}, \\
N_{4}^{2}=B_{1} c_{1}^{2}+2 B_{2} c_{2}^{2}, \quad N_{3}^{2}=B_{1} c_{2}^{2}+2 B_{2} c_{3}^{2}, \quad N_{2}^{2}=B_{1} c_{3}^{2} .
\end{gathered}
$$

Coefficients $S_{7}, S_{6}, S_{5}, S_{4}, S_{3}, S_{2}$ :

$$
\begin{gathered}
S_{7}=\frac{g}{\nu_{2}}\left(\beta_{2} a_{2}^{2}+\gamma b_{2}\right)\left(\frac{b_{2}}{D}-\alpha B_{2}\right) \\
S_{6}=\frac{g}{\nu_{2}}\left[\left(\frac{b_{1}}{D}-\alpha B_{1}\right)\left(\beta_{2} a_{2}^{2}+\gamma b_{2}\right)+4\left(\frac{b_{2}}{D}-\alpha B_{2}\right)\left(\beta_{2} A+\gamma b_{1}\right)\right], \\
S_{5}=\frac{g}{\nu_{2}}\left[\left(\frac{b_{1}}{D}-\alpha B_{1}\right)\left(\beta_{2} A+\gamma b_{1}\right)+3\left(\frac{b_{2}}{D}-\alpha B_{2}\right) c_{1}^{2}\right],
\end{gathered}
$$




$$
\begin{gathered}
S_{4}=\left(\frac{b_{1}}{D}-\alpha B_{1}\right) c_{1}^{2}+2\left(\frac{b_{2}}{D}-\alpha B_{2}\right) c_{2}^{2}, \\
S_{3}=\left(\frac{b_{1}}{D}-\alpha B_{1}\right) c_{2}^{2}+\left(\frac{b_{2}}{D}-\alpha B_{2}\right) c_{3}^{2}, \quad S_{2}=\left(\frac{b_{1}}{D}-\alpha B_{1}\right) c_{3}^{2} .
\end{gathered}
$$

Coefficients $d_{3}^{i}, d_{2}^{i}, d_{1}^{i}$ :

$$
\begin{gathered}
d_{3}^{1}=\rho_{1} g \beta_{1} a_{2}^{1}, \quad d_{2}^{1}=\rho_{1} g \beta_{1} A, \quad d_{1}^{1}=\rho_{1} \nu_{1} c_{1}^{1}, \\
d_{3}^{2}=\rho_{2} g \beta_{2} a_{2}^{2}+\rho_{2} g \gamma b_{2}, \quad d_{2}^{2}=\rho_{2} g \beta_{2} A+\rho_{2} g \gamma b_{1}, \quad d_{1}^{2}=\rho_{2} \nu_{2} c_{1}^{2} .
\end{gathered}
$$

Coefficients $K_{8}^{i}, K_{7}^{i}, K_{6}^{i}, K_{5}^{i}, K_{4}^{i}, K_{3}^{i}, K_{2}^{i}, K_{1}^{i}$ :

$$
\begin{gathered}
K_{8}^{1}=\frac{1}{1008} \frac{\left(g \beta_{1} a_{2}^{1}\right)^{2} \rho_{1}}{\nu_{1} \chi_{1}}, \quad K_{7}^{1}=\frac{1}{144} \frac{\left(g \beta_{1}\right)^{2} \rho_{1}}{\nu_{1} \chi_{1}} A a_{2}^{1}, \\
K_{6}^{1}=\frac{1}{120} \frac{g \beta_{1} \rho_{1}}{\chi_{1}}\left(\frac{g \beta_{1}(A)^{2}}{\nu_{1}}+3 a_{2}^{1} c_{1}^{1}\right), \quad K_{5}^{1}=\frac{1}{24} \frac{g \beta_{1} \rho_{1}}{\chi_{1}}\left(A c_{1}^{1}+2 a_{2}^{1} c_{2}^{1}\right) \\
K_{4}^{1}=\frac{1}{6} \frac{g \beta_{1} \rho_{1}}{\chi_{1}}\left(A c_{2}^{1}+a_{2}^{1} c_{3}^{1}\right), \quad K_{3}^{1}=\frac{1}{2} \frac{g \beta_{1} \rho_{1}}{\chi_{1}} A c_{3}^{1}, \quad K_{2}^{1}=g \beta_{1} \rho_{1} c_{4}^{1}, \quad K_{1}^{1}=g \beta_{1} \rho_{1} c_{5}^{1} \\
K_{8}^{2}=\frac{1}{1008} \frac{g^{2} \rho_{2}}{\nu_{2}}\left(\beta_{2} a_{2}^{2}+\gamma b_{2}\right)\left(B_{2}\left(\beta_{2}-\alpha \gamma\right)+\frac{\gamma b_{2}}{D}\right) \\
K_{7}^{2}=\frac{1}{720} \frac{g^{2} \rho_{2}}{\nu_{2}}\left[\left(\beta_{2} a_{2}^{2}+\gamma b_{2}\right)\left(B_{1}\left(\beta_{2}-\alpha \gamma\right)+\frac{\gamma b_{1}}{D}\right)+\right. \\
\left.+4\left(\beta_{2} A+\gamma b_{1}\right)\left(B_{2}\left(\beta_{2}-\alpha \gamma\right)+\frac{\gamma b_{2}}{D}\right)\right], \\
\frac{1}{120} g \rho_{2}\left[\frac{g}{\nu_{2}}\left(\beta_{2} A+\gamma b_{1}\right)\left(B_{1}\left(\beta_{2}-\alpha \gamma\right)+\frac{\gamma b_{1}}{D}\right)+3\left(B_{2}\left(\beta_{2}-\alpha \gamma\right)+\frac{\gamma b_{2}}{D}\right) c_{1}^{2}\right] \\
K_{5}^{2}=\frac{1}{24} g \rho_{2}\left[\left(B_{1}\left(\beta_{2}-\alpha \gamma\right)+\frac{\gamma b_{1}}{D}\right) c_{1}^{2}+2\left(B_{2}\left(\beta_{2}-\alpha \gamma\right)+\frac{\gamma b_{2}}{D}\right) c_{2}^{2}\right] \\
K_{4}^{2}=\frac{1}{6} g \rho_{2}\left[\left(B_{1}\left(\beta_{2}-\alpha \gamma\right)+\frac{\gamma b_{1}}{D}\right) c_{2}^{2}+\left(B_{2}\left(\beta_{2}-\alpha \gamma\right)+\frac{\gamma b_{2}}{D}\right) c_{3}^{2}\right] \\
K_{3}^{2}=\frac{1}{2} g \rho_{2}\left(B \beta_{1}\left(\beta_{2}-\alpha \gamma\right)+\frac{\gamma b_{1}}{D}\right) c_{3}^{2}, \quad K_{2}^{2}=g \beta_{2} \rho_{2} c_{4}^{2}+g \gamma \rho_{2} c_{6}^{2}, \quad K_{1}^{2}=g \beta_{2} \rho_{2} c_{5}^{2}+g \gamma \rho_{2} c_{7}^{2}
\end{gathered}
$$

Here, $B_{1}=\frac{D A-\chi_{2} \delta b_{1}}{D \chi_{2}(1-\alpha \delta)}, B_{2}=\frac{D a_{2}^{2}-\chi_{2} \delta b_{2}}{D \chi_{2}(1-\alpha \delta)}$.

V. B. Bekezhanova and I. A. Shefer are thankful for the support provided by the Krasnoyarsk Mathematical Center and financed by the Ministry of Science and Higher Education of the Russian Federation in the framework of the establishment and development of regional Centers for Mathematics Research and Education (Agreement no. 075-02-2020-1631). The work of O.N. Goncharova was carried out in accordance with the State Assignment of the Russian Ministry of Science and Higher Education entitled "Modern methods of hydrodynamics for environmental management, industrial systems and polar mechanics" (Govt. contract code: FZMW2020-0008) 


\section{References}

[1] V.B.Bekezhanova, O.N.Goncharova, Problems of the evaporative convection (review), Fluid Dyn., 53(2018), no. 1, S69-S102.

[2] O.N.Goncharova, E.V.Rezanova, Yu.V.Lyulin, O.A.Kabov, Analysis of a convective fluid flow with a concurrent gas flow with allowance for evaporation, High Temperature, 55(2017), no. $6,887-897$.

[3] V.B.Bekezhanova, O.N.Gocnahrova, I.A.Shefer, Analysis of an exact solution of problem of the evaporative convection (Review). Part I. Plane case, Journal of Siberian Federal University. Mathematics \& Physics, 11(2018), no. 2, 178-190.

DOI: 10.17516/1997-1397-2018-11-2-178-190.

[4] V.B.Bekezhanova, O.N.Goncharova, Influence of the Dufour and Soret effects on the characteristics of evaporating liquid flows Int. J. Heat Mass Transfer, 154(2020), art. 119696. DOI: $10.1016 /$ j.ijheatmasstransfer.2020.119696

[5] L.G.Napolitano, Plane Marangoni-Poiseuille flow two immiscible fluids, Acta Astronaut, 7(1980), 461-478.

[6] M.I.Shliomis, V.I.Yakushin, Convection in a two-layers binary system with an evaporation, Collected papers: Uchenye zapiski Permskogo Gosuniversiteta, seriya Gidrodinamika, 4(1972), 129-140 (in Russian).

[7] O.N.Goncharova, E.V.Rezanova, Example of an exact solution of the stationary problem of two-layer flows with evaporation at the interface, J. Appl. Mech. Tech. Phys., 55(2014), no. 2, 247-257. DOI: $10.1134 /$ S002189441402007

[8] O.N.Goncharova, O.A.Kabov, Investigation of the two-layer fluid flows with evaporation at interface on the basis of the exact solutions of the 3D problems of convection, J. Phys.: Conf. Ser., 754(2016), art. 032008.

[9] L.D.Landau, E.M.Lifshitz, Course of Theoretical Physics, Vol. 6, Fluid Mechanics (2nd ed.), Butterworth-Heinemahh, Oxford, 1987.

[10] S.R. De Groot, P.Mazur, Non-equilibrium Thermodynamics, Dover, London, 1984.

[11] V.B.Bekezhanova, O.N.Goncharova, Modeling of three dimensional thermocapillary flows with evaporation at the interface based on the solutions of a special type of the convection equations Appl. Math. Model., 62(2018) 145-162. DOI: 10.1016/j.apm.2018.05.021

[12] V.K.Andreev, Yu.A.Gaponenko, O.N.Goncharova, V.V.Pukhnachev, Mathematical Models of Convection, De Gruyter, Berlin, Boston, 2020.

[13] B.Haut, P.Colinet, Surface-tension-driven instability of a liquid layer evaporating into an inert gas, J. of Colloid and Interface Science, 285(2005), 296-305.

[14] H.Machrafi, Y.Lyulin, C.S.Iorio, O.Kabov, P.C.Dauby, Numerical parametric study of the evaporation rate of a liquid under a shear gas flow: experimental validation and the importance of confinement on the convection cells and the evaporation rate, Int. J. Heat Fluid Flow, 72(2018) 8-19. 


\title{
Решение задачи о двухслойном течении с неоднородным испарением на термокапиллярной границе раздела
}

\author{
Виктория Б. Бекежанова \\ Институт вычислительного моделирования СО РАН \\ Красноярск, Российская Федерация \\ Ольга Н. Гончарова \\ Алтайский государственный университет \\ Барнаул, Российская Федерация \\ Илья А.Шефер \\ Сибирский федеральный университет \\ Красноярск, Российская Федерация
}

\begin{abstract}
Аннотация. В рамках математической модели, учитывающей испарение на межфазной границе и влияние прямого и обратного термодиффузионных эффектов, строится аналог решения Остроумова-Бириха для уравнений термоконцентрационной конвекции. Полученное решение интерпретируется как решение, описывающее установившееся течение испаряющейся жидкости, увлекаемой спутным газопаровым потоком, на рабочем участке плоского горизонтального канала. Приведены точные представления искомых функций. Описан алгоритм определения констант и параметров, которые содержит решение, выписан их явный вид. Решение построено для случая абсорбции пара на верхней стенке канала, которое задаётся граничным условием первого рода для функции концентрации пара. Кратко обсуждается область применимости полученного решения.
\end{abstract}

Ключевые слова: математическая модель, краевая задача, точное решение, испарительная конвекция. 